\title{
C-C chemokine-encoding DNA vaccines enhance breakdown of tolerance to their gene products and treat ongoing adjuvant arthritis
}

\author{
Sawsan Youssef, ${ }^{1}$ Gila Maor, ${ }^{2}$ Gizi Wildbaum, ${ }^{1}$ Nir Grabie, ${ }^{1}$ Alumit Gour-Lavie, ${ }^{1}$ \\ and Nathan Karin 1,3 \\ ${ }^{1}$ Department of Immunology, \\ ${ }^{2}$ Department of Morphological Sciences, and \\ ${ }^{3}$ Rappaport Family Institute for Research in the Medical Sciences and Bruce Rappaport Faculty of Medicine, \\ Technion, Haifa, Israel \\ Address correspondence to: Nathan Karin, Department of Immunology, Technion, PO Box 9649, Haifa 31096, Israel. \\ Phone: 972-4-829-5232; Fax: 972-4-851-7797; E-mail: nkarin@tx.technion.ac.il.
}

Received for publication December 9, 1999, and accepted in revised form June 27, 2000.

\begin{abstract}
Depending on the method of immunization, a single administration of CFA may result in the development of a local inflammatory process or chronic polyadjuvant-induced arthritis (AA). We administered naked DNA vaccines encoding MIP- $1 \alpha$, MCP-1, MIP-1 $\beta$, and RANTES to Lewis rats and confirmed that each of these vaccines induced immunological memory to the corresponding gene product. Upon induction of disease, this memory effectively inhibited the development of the autoimmune condition. Self-specific Ab's developed in DNA-vaccinated animals were neutralizing in vitro and could adoptively transfer the beneficial effect of each vaccine. Repeated administration of the constructs encoding MCP-1, MIP-1 $\alpha$, or RANTES inhibited the development and progression of AA, even when each vaccine was administered only after the onset of disease. This suggests a highly effective way by which the immune system could be re-educated to generate protective immunity against its own harmful activities.
\end{abstract}

J. Clin. Invest. 106:361-371 (2000).

\section{Introduction}

Rheumatoid arthritis (RA) is an inflammatory disorder characterized by infiltration of leukocytes into the synovial tissue (ST) and synovial fluid (SF) of the joints (1). Depending on the method of immunization, a single administration of CFA may result in the development of a local inflammatory process or chronic polyadjuvant-induced arthritis (AA), which histologically and clinically resembles human RA (2). In both diseases proinflammatory cytokines and chemokines are believed to play a pivotal role in the attraction of leukocytes to the site of inflammation and in the initiation and progression of the inflammatory process (for a general review see ref. 3 ). The role of proinflammatory cytokines in disease manifestation has been studied intensively in experimental models and explored in clinical trials (4-9). Recently, the role of chemokines in the regulation of disease has been the focus of several laboratories, including ours.

Chemokines are chemoattractants that mediate leukocyte attraction and recruitment at the site of inflammation (for recent reviews see refs. 10-12). Based on the positions of the first two cysteines, the chemokines can be divided into four highly conserved but distinct supergene families C-C, C-X-C, C, and $\mathrm{C}-\mathrm{X} 3-\mathrm{C}$. The $\mathrm{C}-\mathrm{C}$ family is primarily involved in the activation of endothelium and chemoattraction of $\mathrm{T}$ cells and monocytes to the site of inflammation. The protective competence of anti-C-C chemokinebased immunotherapy has been demonstrated in an experimental model for multiple sclerosis, experimental autoimmune encephalomyelitis (EAE), and AA. Karpus et al. blocked EAE in mice by immunizing them with rabbit anti-mouse polyclonal Abs against macrophage inflammatory protein- $1 \alpha$ (MIP$1 \alpha)(13)$. Gong et al. used an antagonist of monocyte chemoattractant protein 1 (MCP-1) to inhibit arthritis in the MRL-lpr mouse model (14). More recently, Barnes et al. used anti-human RANTES to ameliorate AA in the Lewis rat (15).

We have recently demonstrated that repeated administrations of DNA vaccines encoding proinflammatory cytokines and chemokines such as TNF- $\alpha$, MCP-1, MIP- $1 \alpha$, MIP- $1 \beta$, and RANTES may lead to a breakdown of tolerance to their gene products, resulting in the generation of self-specific neutralizing Ab's that neutralize the in vivo function of the product of each inserted gene $(16,17)$. Thus far, we have explored the above strategy only in EAE. A major disadvantage of this experimental model in the Lewis rat is that both the active and the transferred form of disease manifest only a short-term phase of a transient form of disease, thus rendering this model impractical for experiments in which DNA vaccines are administered for the first 
time only after the onset of disease. Under our working conditions, Lewis rats manifest a long-term AA that includes an acute phase and a long-lasting (more than 100 days) chronic phase of disease. The current study explores, we believe for the first time, chemokine-based naked DNA vaccination in AA and its therapeutic implications.

\section{Methods}

Rats. Female Lewis rats, approximately 6 weeks old, were purchased from Harlan Laboratories Ltd. Israel (Jerusalem, Israel) and maintained under clean conditions in our animal facility.

Induction of $A A$. AA was actively induced, as we described in detail elsewhere (18). Severity of the disease
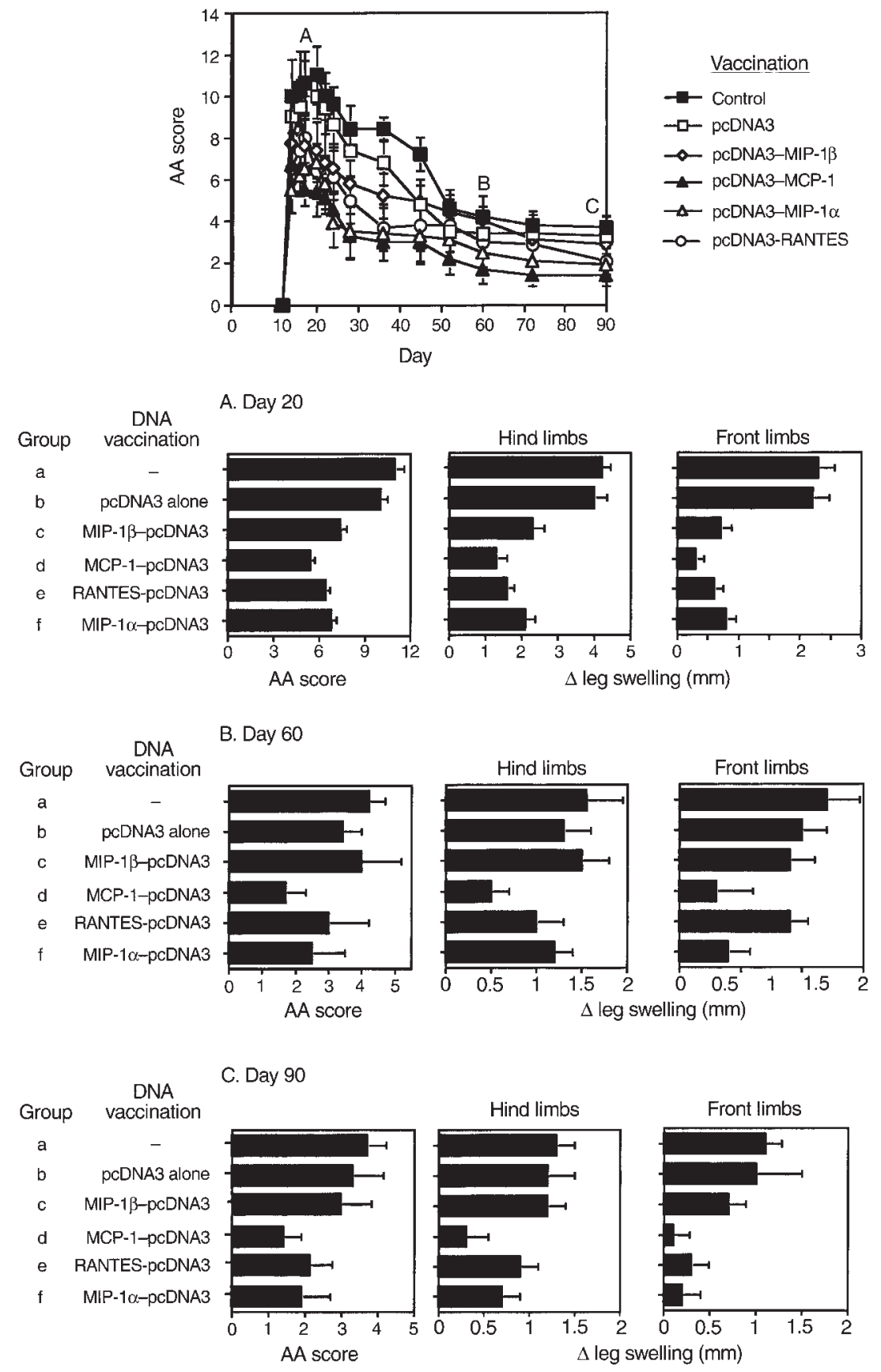

Figure 1

Prevention of AA by C-C chemokine-targeted DNA vaccines. Six groups of ten Lewis rats were exposed to four weekly administrations of various naked DNA vaccines including MCP-1, MIP-1 $\alpha$, MIP-1 $\beta$, and RANTES. Control rats were injected with either the pcDNA3 vector alone or with PBS. Three weeks after the last immunization, all rats were immunized with CFA to induce active AA. AA was scored (daily during the acute phase of disease, weekly during its chronic phase) by an observer blind to the experimental procedure. Leg swelling was also determined. On day 30, four representative rats per group were sacrificed and joints were removed for histological analysis (see Figure 2 and Table 1). Clinical scores and differences in leg swelling are show as mean of ten rats (days 10-30) or 6 rats (day 31-on) \pm SE. 
was quantified by scoring each limb on a scale of $0-4$, which indicates the severity of peripheral joint swelling and erythema: 0 = no signs of disease; 1 = disease evident in a small number of distal joints of the limb; $2=$ disease evident in all of distal joints of the limb; $3=$ disease evident in all of the limb; and $4=$ severe disease evident in all of the limb. The arthritic clinical score was determined as the sum of the scores of all four limbs from each animal (scores from 0-16). The degree of arthritis, indicated by swelling, was quantified by measuring frontlimb and hindlimb circumference using a caliper (Lange Skinfold Caliper; Cambridge Scientific Industries, Cambridge, Massachusetts, USA). Measurements were taken at three time points during the course of disease: days 20,60, and 90 , by an observer blind to the experimental protocol. Measurements are presented as the average of the difference between swelling diameter of treated joints and healthy ones.

$D N A$ vaccination. DNA vaccination was performed using the construct and method we described in detail elsewhere (17). In addition to C-C chemokine-encoding DNA vaccine (17) we have used constructs encoding fractalkine (C-X3-C) and soluble $\beta$-actin (cytoplasmic form). These constructs were obtained using oligonucleotide primers that we have designed according to the published sequence of each gene as follows: fractalkine, sense 5'-ATGGCTCCCTCACAGCTCGCG-3', anti-sense 5'-ACTACCATTTCTAGTCAGGGC-3'; soluble $\beta$-actin (cytoplasmic form), sense 5'-ATGGATGACGATATCGCTGCGCTC-3', anti-sense 5'-CTACCGGCCAGCCAGACG-3'. Each PCR product was cloned and sequence has been verified before being ligated into the pcDNA3 mammalian vector to be used as a DNA vaccine.

Purification of $A b$ 's. $A b$ 's from rat sera were purified using a High-Trap protein G column (Pharmacia Biotech, Piscataway, New Jersey, USA), according the manufacturer's protocol. Then, Ab titer to various chemokines was determined by an ELISA assay as described below. Before being tested for their in vivo characteristics (i.e., ability to affect the course of AA), sera from all DNA-vaccinated rats were subjected to an additional step of purification. Commercially available (Chemicon International, Temecula, California, USA) recombinant MCP-1, MIP-1 $\alpha$, MIP-1 $\beta$, or RANTES was bound to a CNBr-activated Sepharose column according to the manufacturer's instructions (catalog number 17-0820-01; Pharmacia Biotech). Specific Ab's to the gene product of each DNA vaccine were (IgG fraction) loaded on the columns, each consisting of the appropriate commercially available $\mathrm{C}$-C chemokine gene product bound to $\mathrm{CNBr}$, and then eluted by an acidic elution buffer (glycine $\mathrm{pH} 2.5$ ). Isotype determination of the purified $A b$ (using ELISA) revealed that purified $A b$ 's are mostly of the IgG2a isotype (data not shown).

In vitro chemotaxis assay. In vitro chemotaxis assay was conducted as described previously (17). The same protocol was also used for the detection of activated T-cell migration $\left(1.2 \times 10^{6}\right.$ cells from our $\mathrm{CD}^{+}$purified pro- tein derivative-specific [PPD-specific] T-cell line) with the modification of using a different pore size $(8 \mu \mathrm{m})$ of polycarbonate filters (Osmonics Laboratory Products, Livermore, California, USA).

Selection of antigen-specific T-cell lines. CD4 ${ }^{+}$PPD-specific Tcell line was selected using the protocol that we used for the selection of MBP-specific T-cell lines (17), with the dif-
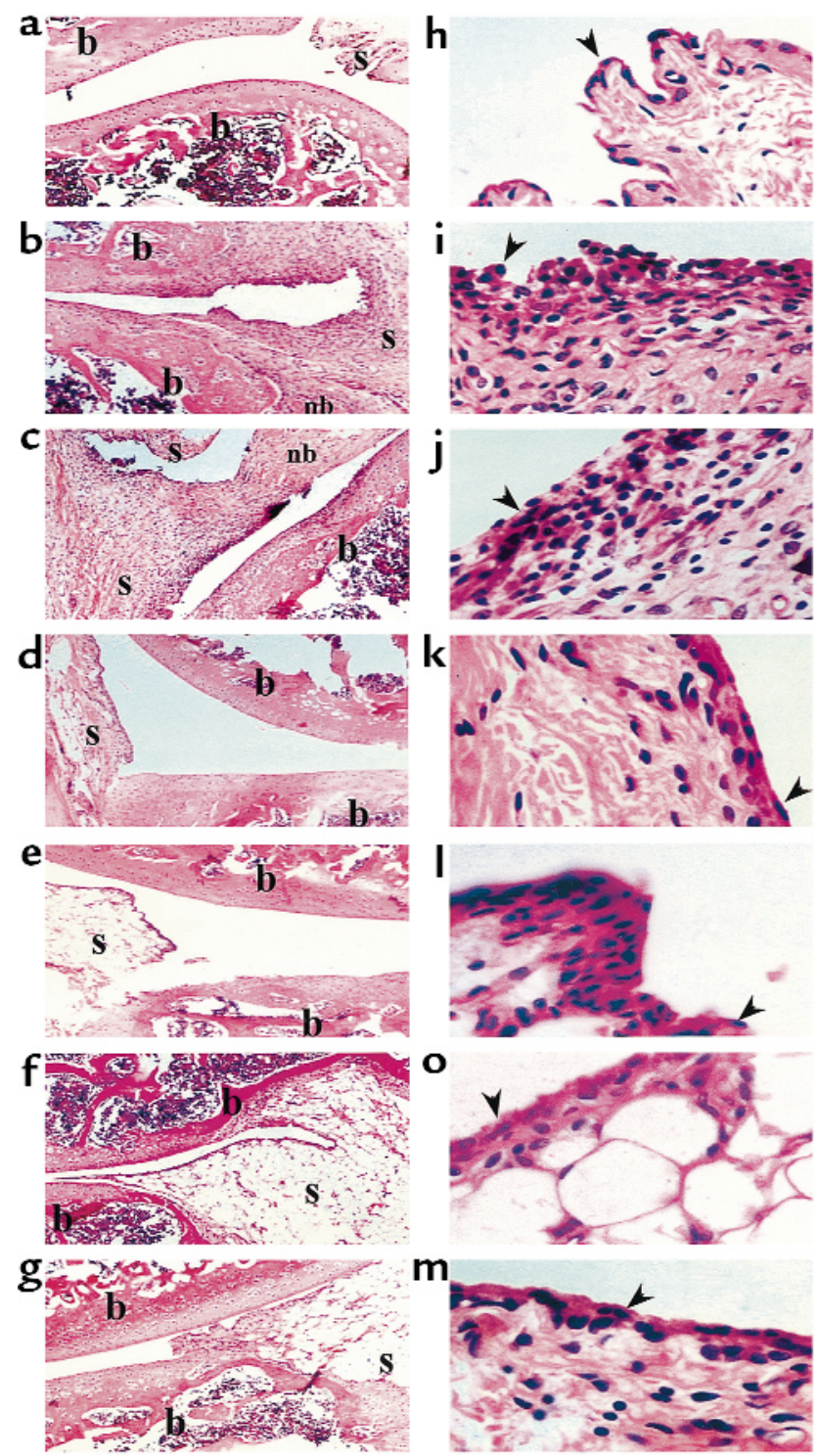

Figure 2

DNA vaccination with C-C chemokines reduces the severity of histological changes in the acute phase of AA in rats. Representative sections from Table 1 are presented. (a-g) Representative synovial joints $(\times 5)$. (h-n) Representative synovial tissue $(\times 40)$. Parts a and $\mathbf{h}$ show naive rat joint; $\mathbf{b}$ and $\mathbf{i}$ show an arthritic joint; $\mathbf{c}$ and $\mathbf{j}$ show joint from rat vaccinated with pcDNA3 vector alone; $\mathbf{d}$ and $\mathbf{k}$ show representative joint from rats vaccinated with MCP- 1 ; e and I show representative joint from rats vaccinated with MIP- $1 \alpha ; \mathbf{f}$ and $\mathbf{m}$ show representative joints from rats vaccinated with MIP-1 $\beta$; $\mathbf{g}$ and $\mathbf{n}$ show representative joints from rats vaccinated with RANTES. The arrowheads point to the synovial lining. Histological evaluation was done by an observer blind to the experimental protocol. b, bone; nb, new bone formation; s, synovial membrane. 


\section{Table 1}

DNA vaccination with $\mathrm{C}-\mathrm{C}$ chemokines reduces the severity of histological changes in the acute phase of AA in rats

\begin{tabular}{lcc}
\hline DNA vaccine & AA induction & Histological score \\
- & - & 0 \\
- & + & $3 \pm 0^{\mathrm{A}}$ \\
PCDNA3 alone & + & $2.8 \pm 0.18^{\mathrm{A}}$ \\
MCP-1 & + & $0.83 \pm 0.33^{\mathrm{D}}$ \\
MIP-1 $\alpha$ & + & $1.3 \pm 0.23^{\mathrm{C}}$ \\
MIP-1 $\beta$ & + & $2 \pm 0.28^{\mathrm{B}}$ \\
RANTES & + & $1.5 \pm 0.24^{\mathrm{C}}$
\end{tabular}

Thirty days after the induction of disease, representative rats (four rats per group) of AA rats subjected previously to various naked DNA vaccines (as described in Figure 1) were histologically analyzed. Histological score was determined on a scale of $0-3$ as explained in Methods. Mean of 12 sections \pm $\mathrm{SE}$ is shown. $P<0.001$ for the comparison of $\mathrm{D}$ with $\mathrm{A} ; P<0.05$ for the comparison of $\mathrm{D}$ with $\mathrm{B} ; P<0.002$ for the comparison of $\mathrm{C}$ with $\mathrm{A} ; P<0.05$ for the comparison of $B$ with $A$.

to a significant $(P<0.01)$ reduction in the severity of the acute phase of disease (on day 20, $6.8 \pm 0.93,5.1 \pm$ $0.7,6 \pm 1.2$, and $6.8 \pm 1.3$ for treatment with MIP- $1 \alpha$, MCP-1, RANTES, and MIP-1 $\beta$, respectively, versus 11 \pm 1.39 and $10 \pm 1.1$ in rats treated with PBS or pcDNA3 alone, respectively). However, during the chronic phase of disease (day 60 and 90) DNA vaccines encoding MIP-1 $\alpha$, MCP-1, RANTES, but not MIP-1 $\beta$, were protective $(P<0.01$ compared with each control group). Representative joint sections from all experimental groups (four animals per group) were obtained on day 30 and screened for histological inflammatory mononuclear cell infiltrate in the synovial membrane, thickness of the synovial lining, joint-space narrowing, and periosteal new bone formation. Histological scores are summarized in Table 1. Representative sec- tions are also presented in Figure 2. At this time, all four detected vaccines led to a significant reduction $(P$ $<0.05)$ in the histological manifestation of disease. Yet, among the C-C chemokine DNA-vaccinated rats, those subjected to the MCP-1 DNA construct manifested the lowest histological score, which was significantly reduced not only from each control group $(P<$ $0.01)$, but also from MIP-1 $\beta$ DNA-vaccinated rats $(P<$ $0.05)$, which excreted the moderated effect of all detected chemokines. A similar pattern of results was obtained during the chronic phase of disease (Figure 1). Taken together, our results show that $\mathrm{C}-\mathrm{C}$ chemokine DNA vaccines can be used effectively to prevent AA. They also demonstrate that whereas the MCP-1-encoding DNA vaccine is the most potent inhibitor of disease, the construct encoding MIP-1 $\beta$ is the less effective one, particularly during the chronic phase of disease.

Self-specific Ab's developed in DNA-vaccinated rats are neutralizing, capable of inbibiting delayed-type bypersensitivity response in vivo and of transferring the protective effect of each vaccine. DNA vaccination can elicit potentially both cellular and humoral responses against products of a given construct (21-25). We have assessed the contribution of the humoral response to the state of AA resistance that follows DNA vaccination. At first, DNA-vaccinated AA rats were followed for the production of self-specific Ab's to the gene product of each DNA vaccine. Our results show, we believe for the first time, that even without being subjected to naked DNA vaccination, rats that developed poly-arthritis (but not a local DTH response resulting from a footpad administration of CFA) displayed a significant $(P$ $<0.05)$ appearance of self-specific $\mathrm{Ab}$ titer to proin-

Table 2

Blockade of leukocyte chemotaxis by C-C chemokine-specific Ab's generated in DNA-vaccinated rats

Purified Ab's (IgG) from:

Chemoattractant:

AA rats

$\begin{array}{cc}\text { PcDNA3 } & \text { MIP-1 } \alpha \\ \text { DNA } & \text { DNA } \\ \text { vaccinated } & \text { vaccinated } \\ \text { AA rats } & \text { AA rats }\end{array}$

Chemotaxis of activated macrophages

Medium

fMLP

MIP- $1 \alpha$

MCP-1

MIP-1 $\beta$

RANTES

$48 \pm 6$
$160 \pm 19$
$148 \pm 18$
$123 \pm 25$
$137 \pm 19$
$144 \pm 22$

Chemotaxis of an activated T-cell line

$\begin{array}{lcr}\text { Medium } & 60 \pm 8 & 54 \pm 10 \\ \text { fMLP } & 190 \pm 15 & 159 \pm 21 \\ \text { MIP-1 } \alpha & 165 \pm 18 & 149 \pm 17 \\ \text { MCP-1 } & 146 \pm 12 & 141 \pm 12 \\ \text { MIP-1 } \beta & 132 \pm 17 & 129 \pm 12 \\ \text { RANTES } & 154 \pm 13 & 146 \pm 11\end{array}$

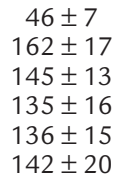

$50 \pm 12$
$180 \pm 23$
$150 \pm 12$
$125 \pm 17$
$135 \pm 18$
$146 \pm 10$

$49 \pm 10$

$158 \pm 18$

$34 \pm 6^{\mathrm{B}}$

$110 \pm 9$

$125 \pm 6$

$142 \pm 14$

$50 \pm 12$
$190 \pm 12$
$155 \pm 18$
$144 \pm 11$
$135 \pm 18$
$143 \pm 15$

$50 \pm 10$

$209 \pm 14$

$48 \pm 11^{\mathrm{B}}$

$137 \pm 9$

$130 \pm 9$

$145 \pm 14$

MCP-1
DNA
vaccinated
AA rats

AA rats

$49 \pm 7$
$153 \pm 13$
$89 \pm 11^{\mathrm{A}}$
$30 \pm 7^{\mathrm{B}}$
$131 \pm 15$
$143 \pm 16$

$143 \pm 16$

$61 \pm 9$
$137 \pm 20$
$79 \pm 19^{A}$
$44 \pm 11^{B}$
$143 \pm 12$
$154 \pm 17$

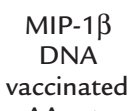

\section{RANTES \\ DNA \\ vaccinated \\ AA rats}

Self-specific Ab's obtained in DNA-vaccinated AA rats (Figure 4) were evaluated for their competence to inhibit the migration of oil-induced peritoneal macrophages or PPD-activated CD4+ line T cells in a Boyden chemotaxis chamber assay. Cells $\left(1.2 \times 10^{6}\right.$, either macrophages or T cells) were added to the upper well. Commercially available (Chemicon) MIP- $1 \alpha(200 \mathrm{ng} / \mathrm{mL})$, MCP- 1, MIP- $1 \beta$, and RANTES (100 ng/mL each) were used as chemoattractants. fMLP (10-7M; Sigma Chemical Co., St. Louis, Missouri, USA) was used as a positive control for chemoattraction. Purified Ab's (IgG purification) were added at a concentration of $10 \mu \mathrm{g} / \mathrm{mL}$. Results are shown as mean of triplicates $\pm \mathrm{SE}$. ${ }^{\mathrm{P} P}<0.05$. ${ }^{\mathrm{B} P}<0.001$. 
a

\begin{tabular}{|c|c|}
\hline $\begin{array}{c}\text { DNA } \\
\text { vaccination }\end{array}$ & $\begin{array}{c}\text { CFA } \\
\text { immunization }\end{array}$ \\
\hline- & non \\
\hline - & footpad \\
\hline pcDNA3 & non \\
\hline- & base tail \\
\hline pcDNA3 & base tail \\
\hline $\begin{array}{l}\text { pcDNA3-MCP-1 } \\
\text { pcDNA3-MCP- } 1\end{array}$ & $\begin{array}{l}\text { non } \\
\text { footpad }\end{array}$ \\
\hline pcDNA3-MCP-1 & base tail \\
\hline
\end{tabular}

C

\begin{tabular}{|c|c|}
\hline $\begin{array}{l}\text { DNA } \\
\text { raccination }\end{array}$ & $\begin{array}{c}\text { CFA } \\
\text { immunizati }\end{array}$ \\
\hline- & non \\
\hline- & \\
\hline DNA3 & \\
\hline- & \\
\hline pcDNA3 & base tail \\
\hline $\begin{array}{l}\text { pcDNA3-MIP- } \\
\text { pcDNA3-MIP- }\end{array}$ & $\begin{array}{l}\text { non } \\
\text { footpad }\end{array}$ \\
\hline DNA3-MIP & base ta \\
\hline
\end{tabular}

Observed

response

Local DTH

Poly-arthritis $\angle Z Z A$

Poly-arthritis $Z Z Z Z A$

Local DTH

Poly-arthritis

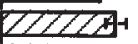

DCDCDA-1

CCZCCCZA-

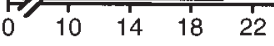

$\log _{2} A b$ titer

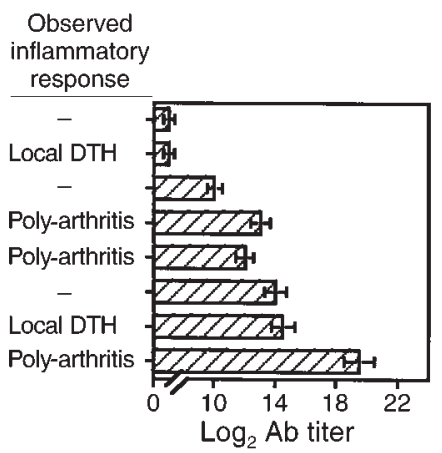

b

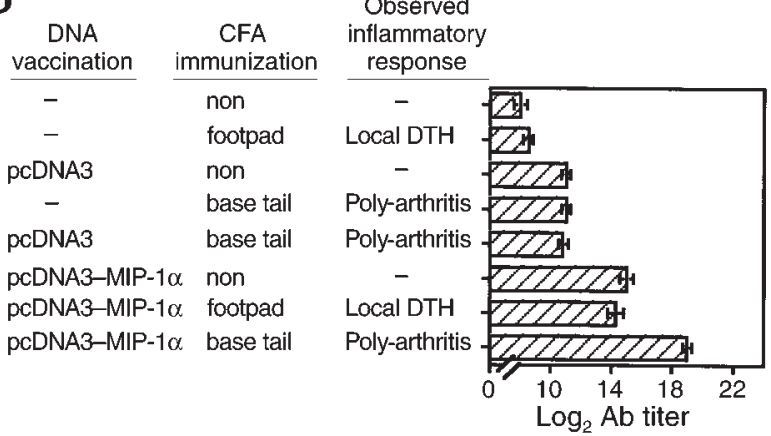

d

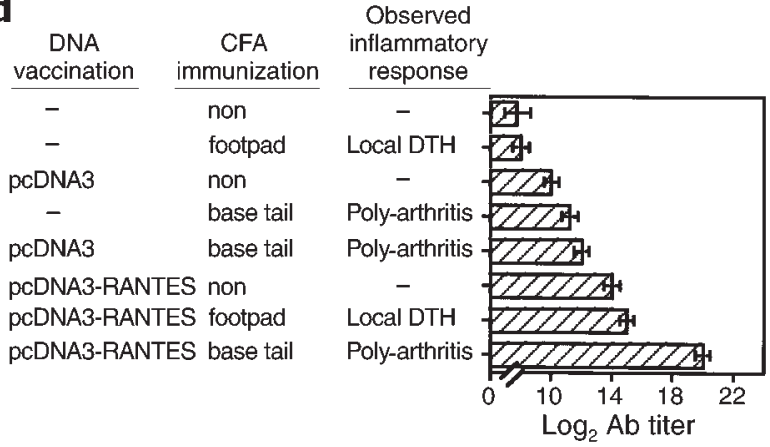

\section{Figure 3}

Breakdown of tolerance to self in DNA-vaccinated rats leads to the production of self-specific Ab's. Lewis rats were subjected to the subsequent administration of various C-C chemokine DNA constructs, as described in Figure 1. Control rats were injected with pcDNA3 alone or with PBS. Three weeks later these rats were separated to subgroups that were administered CFA in two different ways: footpad injection to induce a local DTH response and tail-base administration to induce poly-arthritis. On day 20 anti-self Ab titers in serum were determined by ELISA. Results are shown as mean of three different serum samples $\pm \mathrm{SE}$.

ference of using PPD as a target antigen for selection. After three selection cycles, specificity of the line was verified by an in vitro proliferation assay. At this time FACS analysis confirmed that more than $95 \%$ of the line cells are $\mathrm{CD}^{+}$.

Delayed-type bypersensitivity. Rats were immunized intradermally into the dorsal surface of the ear with 10 $\mu \mathrm{g}$ PPD (Connaught Laboratories, Toronto, Ontario, Canada) in $25 \mu \mathrm{L}$ of PBS (or with PBS alone). Ear thickness was measured using a caliper and determined as the mean of five repeated measurements plus or minus $\mathrm{SE}$. The ear thickness at time 0 was subtracted from the 24-hour measurement to give the amount of delta ear swelling in response to PPD (19).

Evaluation of anti-chemokine Ab titer in sera of DNA-vaccinated rats. A direct ELISA assay has been used to determine the anti-C-C chemokine Ab titer in DNA-vaccinated rats as we described in detail elsewhere (17). Results of experiments conducted in triplicate were calculated as $\log _{2} \mathrm{Ab}$ titer plus or minus SE.

Histopathology. Joints were removed from rats 30 or 90 days after disease induction, fixed with $10 \%$ buffered formalin, decalcified in 5\% ethylenediaminetetraacetic acid in buffered formalin, embedded in paraffin, and sectioned along the midline through the metatarsal region (20). Sections were stained with hematoxylin and eosin and analyzed by a histopathologist who was a blind observer to the experimental procedure. Evalu- ation was made based upon inflammatory mononuclear cell infiltrate in the synovial membrane, thickness of the synovial lining, joint-space narrowing, and periosteal new bone formation. Histological score was determined as follows: $0=$ no evidence of disease; $1=$ mild lymphocytic infiltrate; 2 = widespread mononuclear inflammation and thickening of the synovial lining; and 3 = severe bone destruction, new bone formation, and destruction of the synovial lining (20).

Statistical analysis. Significance of differences was examined using Student's $t$ test. A $P$ value of less than 0.05 was considered to be significant. One-way multiple range ANOVA test with significance level of $P$ values less than 0.05 was performed for multiple comparisons of $\mathrm{Ab}$ titers to various $\mathrm{C}-\mathrm{C}$ chemokines in naked DNA-vaccinated rats.

\section{Results}

Prevention of AA using C-C chemokine naked DNA vaccines. Under our working conditions AA manifests a longlasting form of disease that includes an acute phase, peaking around day 20 , and a chronic phase that persists for more than 90 days (Figure 1). First, we evaluated the ability of various $\mathrm{C}-\mathrm{C}$ chemokine-encoding DNA constructs to prevent AA (i.e., vaccines were administered before the induction of disease). Each of the four C-C chemokine-encoding DNA vaccines led 

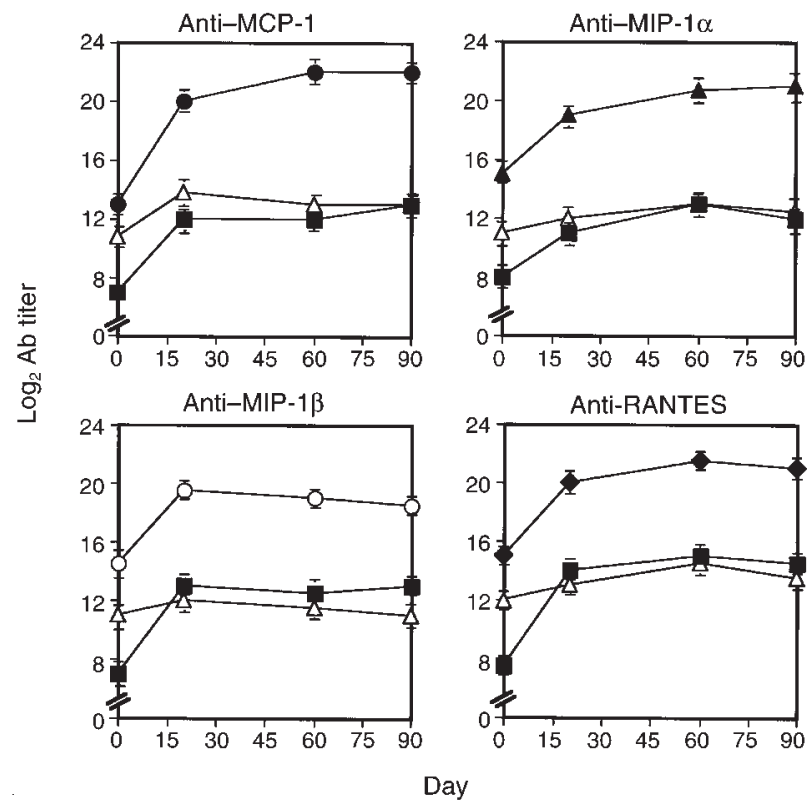

Day

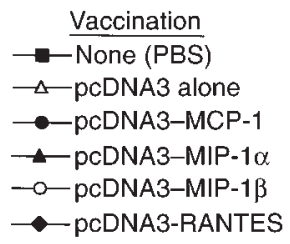

Figure 4

Self-specific Ab's to the gene product of each DNA vaccine are continually produced during the acute and the chronic phase of disease. Lewis rats were subjected to the administration of various C-C chemokine DNA constructs, as described in Figure 1. Control rats were injected with PcDNA3 alone or with PBS. Three weeks later these rats were administered CFA to induce poly-arthritis. At different time points the kinetics of anti-self $A b$ appearance in serum was determined by ELISA. Results are shown as mean of three different serum samples \pm SE.

flammatory chemokines (Figure 3). This notable increase in chemokine-specific titer during AA was, however, not sufficient to prevent the development and progression of the inflammatory condition in $\mathrm{AA}$ (Figure 1). After induction of AA, the chemokine-specific $\mathrm{Ab}$ titer profoundly increased in rats subjected previously to DNA vaccines encoding each relevant gene product (Figure 3; $\log _{2} \mathrm{Ab}$ titer of $20 \pm 0.55,19 \pm$ $0.33,19.5 \pm 0.55$, and $20 \pm 0.47$ to MCP-1, MIP- $1 \alpha$, MIP-1 $\beta$, and RANTES, in MCP-1-, MIP- $1 \alpha-$, MIP- $1 \beta-$, or RANTES-DNA vaccinated rats, respectively. $P<$ 0.001 for the comparison of each Ab titer to the titer developed in AA rats subjected previously to pcDNA3 alone or PBS). Each elevated titer continued to persist during the chronic phase of disease (Figure 4), as did the clinical effect of these vaccines (Figure 1).

Several experiments were then conducted to explore the possible contribution of each $\mathrm{Ab}$ to the high state of resistance obtained in DNA-vaccinated rats (Figure 1). At first, we determined possible cross-reactivity between Ab's from various DNA-vaccinated AA rats
(Figure 5). Then we evaluated the competence of each $\mathrm{Ab}$ to inhibit leukocyte migration in vitro (Table 2), as well as to block DTH to PPD (Figure 6b). Finally, each $\mathrm{Ab}$ was evaluated for its competence to provide a high state of disease resistance when adoptively transferred to AA rats (Figure 6a). Figure 5 shows that C-C chemokine DNA-vaccinated rats develop a highly specific titer against homologous antigen: MCP-1, MIP$1 \alpha$, MIP-1 $\beta$, and RANTES (Figure 5; $P<0.001$ for the comparison of self-specific titer to each gene product compared with the other chemokines). MCP-1 DNA-vaccinated rats, however, exhibited a notable cross-reactive $\mathrm{Ab}$ titer against MIP- $1 \alpha$ (Figure $5 \mathrm{~b}$ ). Then, $A b$ 's were purified (IgG fraction, protein $G$ purification) and evaluated for their ability to inhibit the migration of oil-induced peritoneal macrophages (Table 2) or PPD activated CD $4^{+} \mathrm{T}$ cells (Table 2 ) in a Boyden chemotaxis chamber assay. Self-specific Ab's generated in DNA-vaccinated rats could profoundly block both monocyte and T-cell chemotaxis induced by each relevant chemokine (Table 2) and to a much lesser extent (if at all) chemotaxis induced by antisera from rats previously subjected to DNA vaccination with other C-C chemokine constructs. The acceptance of a partial blockade of chemotaxis mediated by MIP- $1 \alpha$ using antisera from MCP-1 DNA-vaccinated rats could, however, be attributed to the partial cross-reactivity to MIP- $1 \alpha$ in MCP-1 DNA-vaccinated rats as shown in Figure $5 \mathrm{~b}$ and previously (see ref. 17). These Ab's were then evaluated for their ability to provide
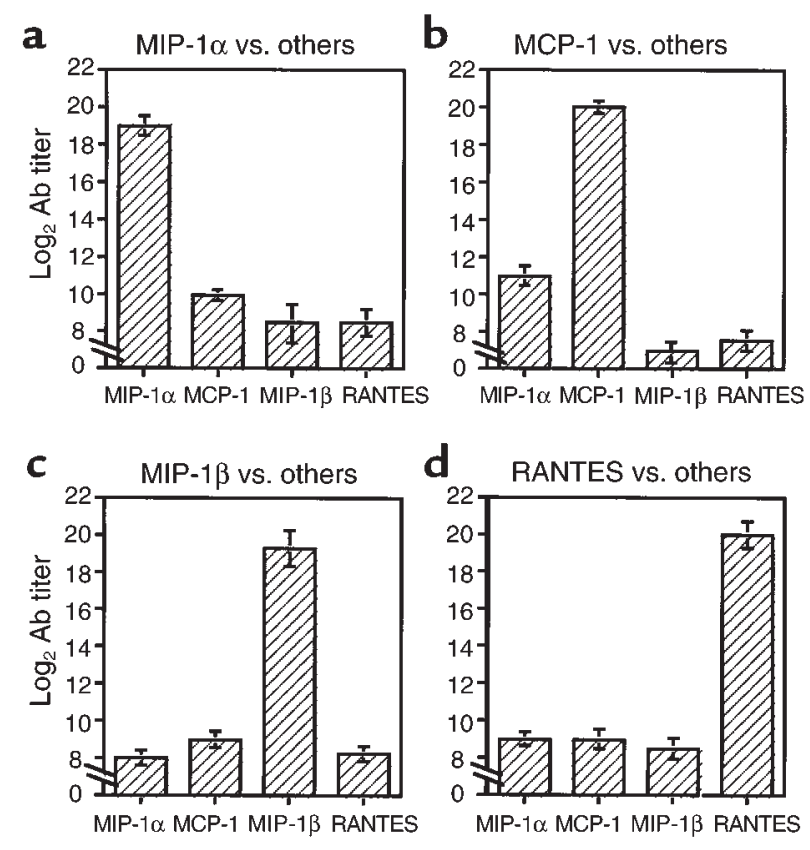

Figure 5

Specificity of anti-chemokine Ab's generated in DNA-vaccinated rats. Self-specific antibodies from DNA vaccinated rats (see Figure 3 ) were evaluated for possible cross-reactivity between the four relevant $C-C$ chemokines: MCP-1, MIP- $1 \alpha$, MIP- $1 \beta$, and RANTES by ELISA. Results are shown as mean of three different sera samples $\pm \mathrm{SE}$. 
a

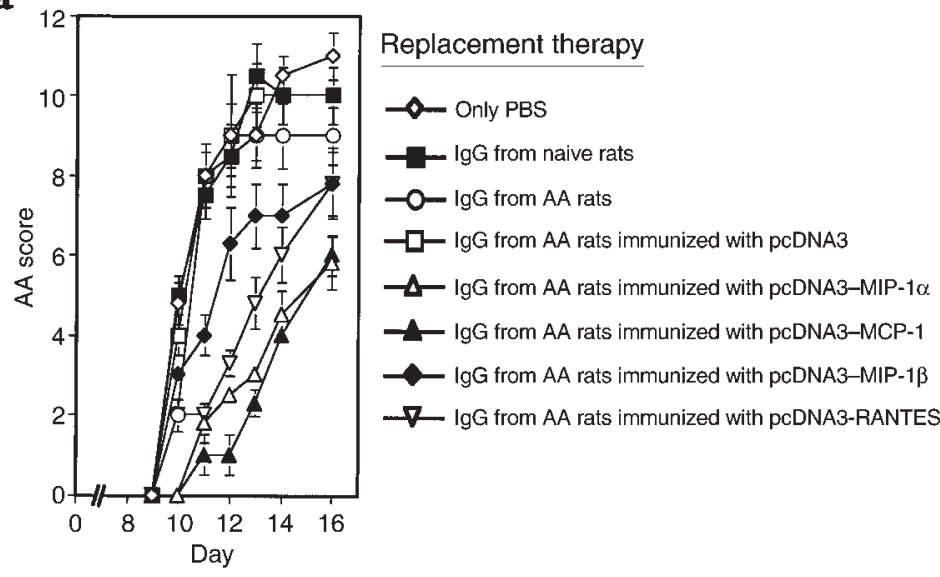

b

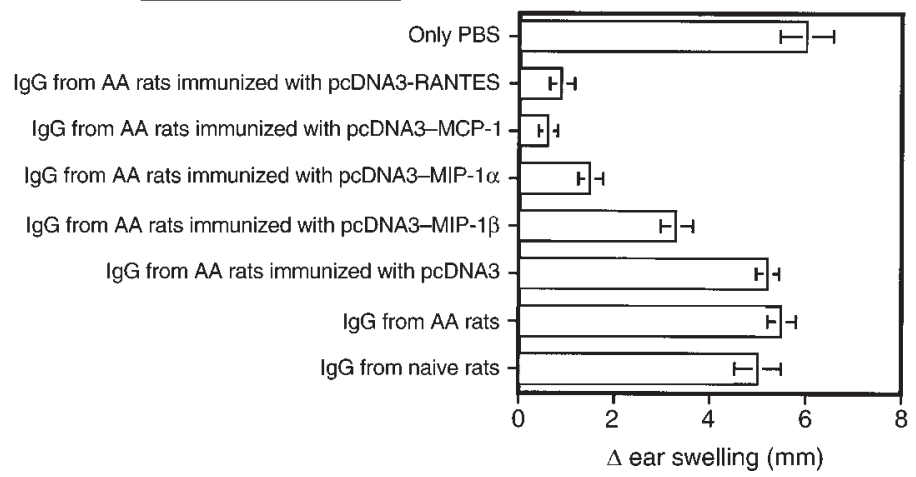

Figure 6

Anti-chemokine Ab's produced by DNA vaccination block DTH response and provide subsequent protection from severe AA. (a) Eight groups of six rats each were immunized with CFA to develop poly-arthritis. Seven, 10, and 12 days after the active induction of disease, these rats were challenged (intravenously) with $200 \mu \mathrm{g}$ of IgG (protein $G$ purification, $\mathrm{CNBr}$ purification) from the different groups described in Figure 3. Control rats were injected with either PBS, IgG from naive rats, IgG from AA or from AA rats previously administered pcDNA3 alone. AA was scored daily by an observer blind to the experimental procedure. Results are shown as mean clinical score of six rats in each group \pm SE. Five to 7 days after the last administration of neutralizing Ab's to each of the above chemokines, disease severity regained the level of control AA rats (not shown). (b) Inhibition of adoptively induced DTH by anti-C-C chemokine Ab's. Lewis rats were intravenously administered $10^{7}$ activated CD4 ${ }^{+}$PPD-specific T cells and were randomly separated into eight groups of five rats each. One hour later each group was subjected to an in vivo administration (intravenously) of various Ab's, as described above (a). Each rat was then immunized intradermally into the dorsal surface of the ear with $10 \mu \mathrm{g}$ PPD in $25 \mu \mathrm{L}$ of PBS (or with PBS alone). Results are presented as mean of delta ear thickness of five different ears \pm SE. protection from severe ongoing AA (Figure 6a) and to inhibit adoptively induced DTH response to PPD (Figure $6 \mathrm{~b}$ ). Before being tested for their in vivo characteristics, sera from all C-C chemokine DNA-vaccinated rats were purified on Sepharose columns, each consisting of the appropriate commercially available C-C chemokine gene product bound to $\mathrm{CNBr}$, as described in Methods. Repeated administration of neutralizing Ab's from MCP-1 and MIP- $1 \alpha$ DNA-vaccinated rats led to a marked reduction in disease severity as compared with all control groups $(P<0.001$ at all time points between the onset of disease and day 16). Except for a single time point (day 16), neutralizing Ab's from RANTES DNA-vaccinated rats also had a beneficial effect on disease manifestation $(P<0.05$ compared with the effect of antisera from pcDNA3 vaccinated rats). Clinical evaluation of each experimental group was also determined by measuring swelling of limbs and by an histological evaluation (not shown). Five to 7 days after the last administration of neutralizing Ab's to each of the above chemokines, disease severity regained the level of control AA rats (not shown). This further emphasizes the advantages of naked DNA vaccination over neutralizing $\mathrm{Ab}$ therapy. In another experiment, rats were administered a CD4 ${ }^{+} \mathrm{T}$-cell line, which we established in response to PPD, and evaluated the in vivo ability of each Ab to inhibit DTH induced by immunization of PPD to the ear (Figure 6b). It appears that Ab's to all three protective chemokines
(MCP-1, MIP-1 $\alpha$, and RANTES), but not to MIP-1 $\beta$, significantly $(P<0.001)$ blocked PPD-induced DTH, as compared with antisera from pcDNA3 AA rats. DTH is dependent on transmigration of antigen-specific $T$ cells and on the activity of macrophages that follow these T cells. Because self-specific Ab's to MCP-1, MIP$1 \alpha$, and RANTES can potentially block the migration of macrophages and activated T cells (Table 2), it is not clear which of the two characteristics contributes more to DTH blockade, as observed in Figure 6b. Nevertheless, these findings may explain, in part, the protective competence of MCP-1-, MIP- $1 \alpha-$, and RANTESencoding, and (to a much lesser extent) MIP-1 $\beta$-encoding, DNA vaccines in AA (Figures 1 and 2 and Table 1).

Treatment of an established disease by naked DNA vaccines encoding $C$-C chemokines. In the first set of experiments, MCP-1-encoding DNA vaccine was evaluated for possible ability to inhibit an ongoing disease (Figure 7a). Lewis rats were immunized with CFA to induce active AA and were separated into three random groups of 12 rats each. Two of these groups were then subjected to three repeated administrations of either pcDNA3 alone or of the MCP- 1 construct ( $300 \mu \mathrm{g}$ per rat) every other day beginning at the onset of disease (day 10). The third group was inoculated with PBS. Whereas all control and pcDNA3-treated rats continued to develop severe AA, those administered with the MCP-1 DNA vaccine exhibited a markedly reduced form of disease (Figure 7a; day 25, mean maximal score of $11 \pm 1$ and 


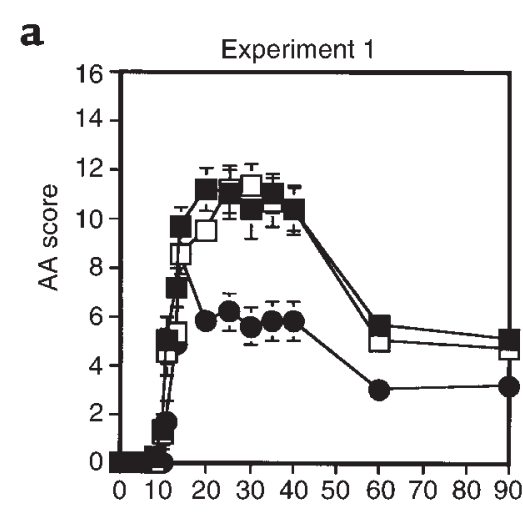

c

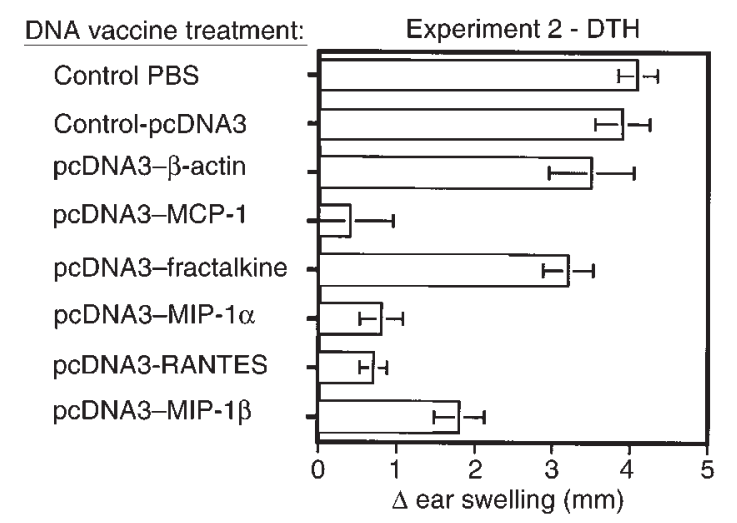

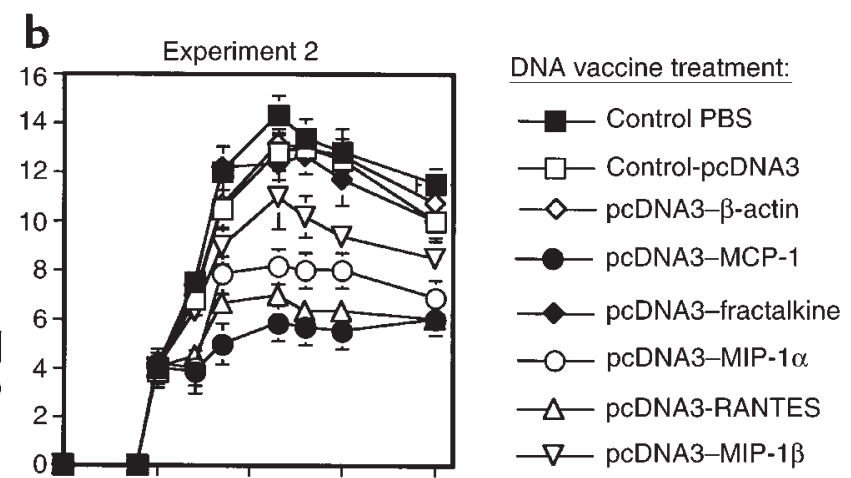

Figure 7

Treatment of established AA by C-C chemokine DNA vaccine. (a) Lewis rats were immunized with CFA to induce active $A A$ and then randomly separated into three groups of 12 rats each. At the onset of disease (day 10) and on days 12 and 14 two of these groups were subjected to three repeated administrations of $300 \mu \mathrm{g}$ per rat of either pcDNA3 alone or of the MCP-1 construct. The third group was inoculated with PBS. In a continuing experiment (b) the same protocol was applied on eight groups of 12 rats each treated with either pcDNA3 alone, RANTES, MCP-1, MIP-1 $\alpha$, MIP-1 $\beta$, fractalkine, and soluble $\beta$-actin (cytoplasm form) constructs. The last group was inoculated with PBS. In both experiments AA was scored daily by an observer blind to the experimental procedure. Results are shown as mean clinical score of 12 rats \pm SE. On day 24 , rats (five per group) were evaluated for their ability to mount a PPD-specific DTH response (c), as described in Figure 6b. Data were determined as mean of five repeated measurements $\pm \mathrm{SE}$.
$11.2 \pm 0.9$ in rats treated with either PBS or pcDNA3 alone versus $6.2 \pm 0.76, P<0.001$, for the comparison of MCP-1 DNA-vaccinated rats to each control group). Representative joint sections from all experimental groups were obtained on day 30 and evaluated for histological analysis of synovitis, cartilage loss, and bone erosion (Figure 8). Sections obtained from AA rats treated with the MCP-1 DNA construct displayed a marked reduction in each of the above parameters as compared with control and pcDNA3-treated AA rats. The beneficial effect of the treatment was long lasting and covered not only the acute phase, but also the chronic phase of disease. Thus, 60 and 90 days after induction of disease, AA rats treated with the MCP-1 DNA construct manifested a significantly reduced form of disease as determined by clinical scores (Figure 7a) and by histological analysis of the joints obtained during the acute (day 30) and chronic (day 90) phase of disease (Figure 8).

In another experiment we used the same experimental protocol to compare the therapeutic ability of DNA constructs encoding each of the various $\mathrm{C}-\mathrm{C}$ chemokines, a chemokine from a different family (the chemokine domain of fractalkine, C-X3-C), and a nonrelevant gene product (the soluble form of $\beta$-actin). It appears that the administration of RANTES-, MCP-1-, or MIP- $1 \alpha$ - but not MIP-1 $\beta$-encoding or C-X3$\mathrm{C}$-encoding DNA vaccines remarkably reduced the severity of ongoing AA (Figure $7 \mathrm{~b} ; P<0.01$ ). Disease inhibition was associated with a reduction in PPD- induced DTH (Figure $7 c ; P<0.001$ ) and with an elevated titer of $A b$ 's to the gene product of each vaccine (data not show), which may explain, in part, the protective competence of these vaccines.

\section{Discussion}

The current study uses naked DNA vaccination to generate protective immunity against self gene products that are associated with leukocyte migration to a site of inflammation (i.e., C-C chemokines) and thus interfere with the development of a $T$ cell-mediated autoimmune disease of the joints. We show here, we believe for the first time, that during the course of AA self-specific Ab's to proinflammatory chemokines are being generated (Figure 3). It is not clear whether these Ab's do manifest a regulatory role in AA. Nevertheless, their appearance in association with disease manifestation but not after footpad administration of the same amount of CFA may suggest that these $\mathrm{Ab}$ 's do manifest a regulatory role in $\mathrm{T}$ cell-mediated autoimmunity. This response is, however, not sufficient to prevent the development and progression of the autoimmune disease developed in these animals. In an attempt to increase this response we thought to administer DNA construct encoding C-C chemokines, together with a repeated immunostimulatory sequence (ISS) $(26,27)$. The rationale was that the addition of this construct might lead to the establishment of a strong immunological memory to the gene product of each vaccine. We show here that after the induction of an autoimmune condition this memory is 
indeed "turned on" (Figure 4) to provide protective immunity (Figure 1). The biological significance of the association between the development of an autoimmune condition at a privileged autoimmune site and the enhancement of anti-self responses against anti-inflammatory cytokines/chemokines that is largely produced at the site of inflammation is apparent. Nevertheless, the underlying mechanism by which the immune system distinguishes a gene product transcribed at a privileged autoimmune site from the same gene product transcribed at a local site of inflammation is still elusive. C.C. Goodnow and colleagues have demonstrated that peripheral clonal exclusion of self-reactive B cells occurs in germinal centers of lymph nodes that drain tissues lacking immune surveillance (i.e., immune-privileged areas), where competition for follicular niches do not exclude self-reactive cells from the recirculating B-cell repertoire (28). In rats immunized in the hind footpads with CFA to develop a local DTH response, the inflam- matory process is indeed limited to the site of immunization, whereas in poly-arthritis an inflammatory process also occurs in the forelimbs, which have never been subjected to CFA immunization. Naked DNA vaccination, however, increases the generation of memory lymphocytes, which probably enables the amplification of such a self-specific immune response. We have shown previously that in C-C chemokine DNA-vaccinated rats developing a short-term autoimmune disease (EAE), selfspecific $\mathrm{Ab}$ titers regress back to background levels after recovery (day 40) $(17,29)$. We now show that in chronic AA these titers proceeded for 90 days or more (Figure 4). This further emphasizes the association between the development and progression of an autoimmune condition at a site with an immune prevalence and the development and maintenance of self-specific immunity to proinflammatory cytokines/chemokines.

Several investigators have already explored neutralizing the activity of chemokines as a way to treat
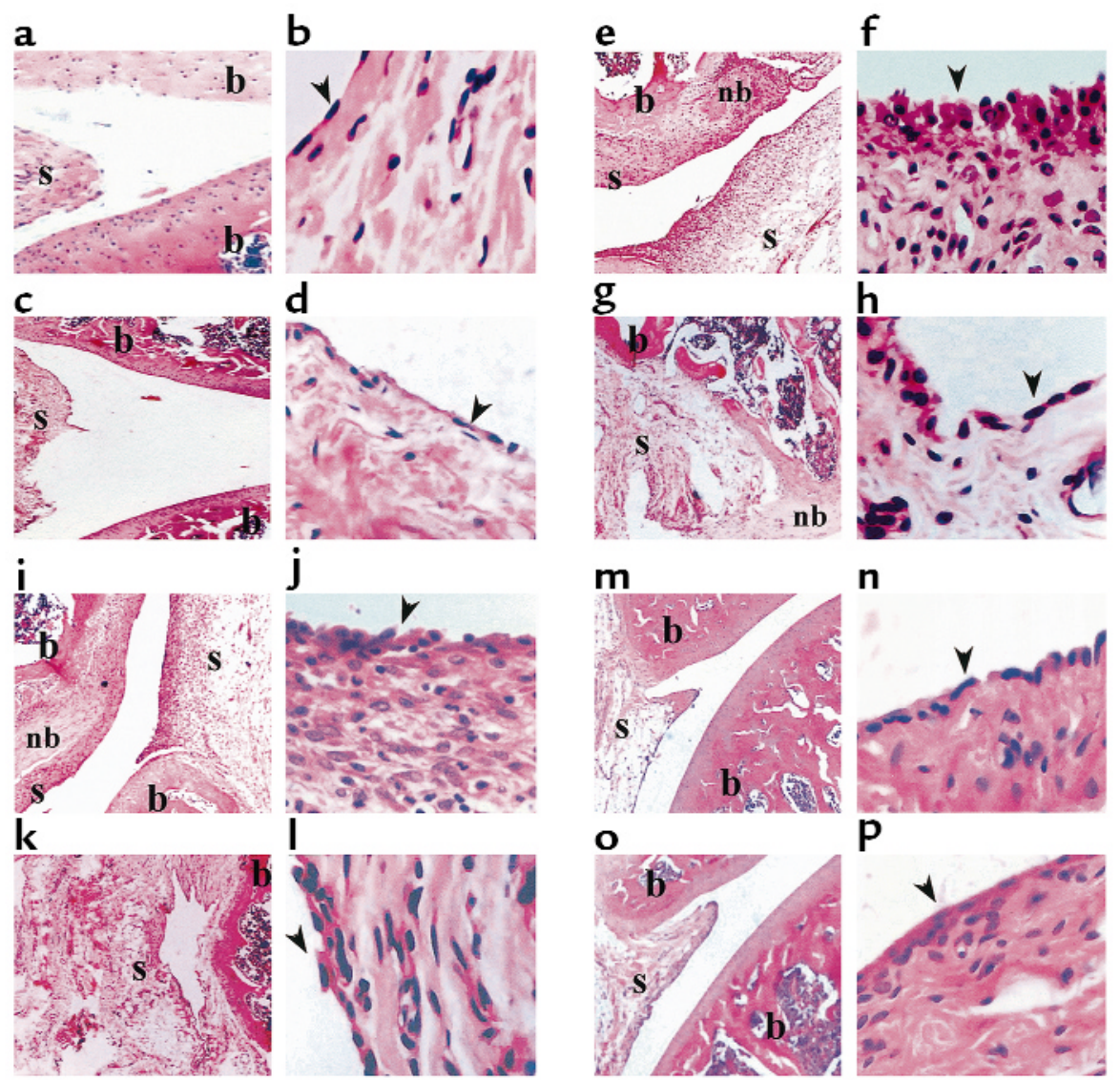

$\mathbf{n}$
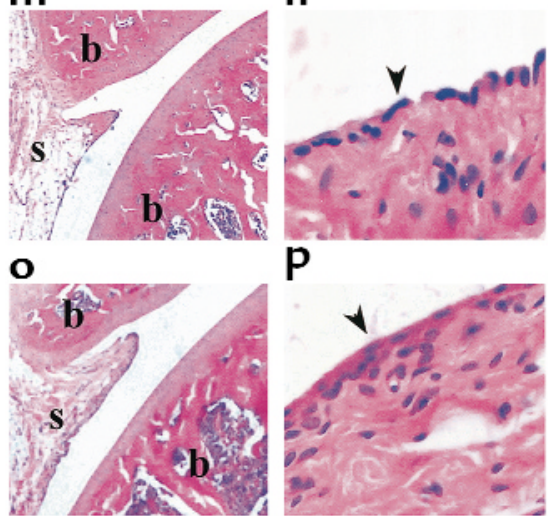

P

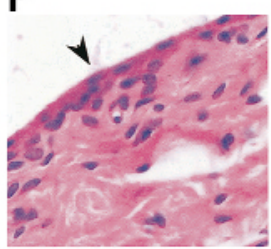

\section{Figure 8}

Inhibition of ongoing AA by MCP-1-targeted DNA vaccination modulates histological changes in the acute and chronic phase of disease. Thirty and 90 days after AA induction joint samples from each of the experimental groups described in Figure 7a (four representative rats per group) were subjected for histological analysis ( 12 sections each group). (a, $\mathbf{c}, \mathbf{e}, \mathbf{g}, \mathbf{i}, \mathbf{k}, \mathbf{m}$, and $\mathbf{o})$ Representative synovial joints ( $\times 5$ ). $(\mathbf{b}, \mathbf{d}, \mathbf{f}, \mathbf{h}, \mathbf{j}, \mathbf{l}, \mathbf{n}$, and $\mathbf{p})$ Representative synovial tissue $(\times 40)$. $\mathbf{a}$ and $\mathbf{b}$ show naive joints taken, with age matching to the experiment rats (day 30); $\mathbf{c}$ and $\mathbf{d}$ show naive joints taken, with age matching to the experiments rats (day 90). $\mathbf{e}$ and $\mathbf{f}$ show arthritic joints taken 30 days after disease induction, and $\mathbf{g}$ and $\mathbf{h}$ arthritic joints taken 90 days after disease induction. $\mathbf{i}$ and $\mathbf{j}$ show pcDNA3-treated joints taken 30 days after disease induction, $\mathbf{k}$ and $\mathbf{I}$ show pcDNA3-treated joints taken 90 days after disease induction. $\mathbf{m}$ and $\mathbf{n}$ show MCP-1-treated joints taken 30 days after disease induction, $\mathbf{o}$ and $\mathbf{p}$ show pcDNA3-treated joints taken 90 days after disease induction. The arrowheads point to the synovial lining. b, bone; nb, new bone formation; s, synovial membrane. 
arthritis. In a very recent study, neutralizing Ab's to epithelial neutrophil-activating peptide 78 (ENA-78) were found capable of inhibiting the development of AA if administered before but not after the onset of disease (30). In another recent study, Barnes et al. used anti-human RANTES to ameliorate AA in the Lewis rat (15). More is known about the role of MCP-1 in the regulation of arthritis. Gong et al. used an antagonist of MCP-1 to inhibit arthritis in the MRL-lpr mouse model (14). In the model of streptococcal cell wall-induced arthritis it has been shown that anti-IL4 and anti-MCP-1 Ab's block the disease (31). The same study demonstrated that neutralizing IL-4 by itself leads to a marked reduction in MCP- 1 mRNA transcription at the autoimmune site and to inhibition of the development of disease. This further implies a role for MCP-1 in arthritis (31). One major disadvantage in treating an autoimmune condition by applying an anti-chemokine/cytokine- or anti-chemokine/cytokine-receptor therapy is that these diseases, in humans are usually chronic. Treating chronic diseases with xenogeneic neutralizing $\mathrm{Ab}$ 's or soluble receptors is very expensive, inconvenient, and may lead to production of Ab's capable of neutralizing these drugs. The therapeutic strategy suggested here has an advantage over the methods discussed above, since it resulted in the generation of immunity to autologous antigens that accelerates during the course of an autoimmune condition in accordance with the disease's progression. This makes this type of therapy an ideal alternative way to treat RA and possibly other $T$ cell-mediated autoimmune diseases $(16,17)$. The second disadvantage of applying anti-chemokine/cytokine immunotherapy in $\mathrm{T}$ cell-mediated autoimmunity is that the treatment is not disease specific and may lead to suppression/alteration of other immunological functions. Indeed, our data show those protective Ab's (anti-MIP-1 $\alpha$, anti-MCP-1, anti-RANTES, and, to a much lesser extent, anti-MIP-1 $\beta$ ) effectively block transferred DTH responses (Figure 6). Along with these observations we show that protective DNA vaccines encoding the genes of these products lead to a significant reduction in PPD-induced DTH response (Figure 7). Once again, the ability to effectively treat AA (Figure $7 b$ ) was associated with the ability to inhibit DTH. That is, MIP-1 $\alpha-$, MCP-1-, and RANTES-encoding DNA vaccines were highly protective and led to a marked reduction in PPD-induced $\mathrm{DTH}$, whereas the MIP-1 $\beta$ DNA vaccine was much less effective in treating AA and also excreted a limited effect on PPD-induced DTH response. Ultimately, an ideal DNA vaccine would excrete a maximal effect on the clinical manifestation of an autoimmune condition with a minimal effect on other immunological functions. The development of a disease-specific chemokine/cytokine-encoding DNA vaccine is dependent on future characterization of organ-specific/disease-specific proinflammatory factors.
Finally, it is well documented that chemokines share receptors (for a recent review see ref. 11). For example, MCP-1, MCP-2, MCP-3, and MCP-4 all bind CCR2, whereas MIP- $1 \alpha$, MIP-1 $\beta$, and RANTES bind CCR5. Thus, it is rational to believe that neutralizing the in vivo function of a single chemokine would lead to a redundancy in the in vivo output. Remarkably, in vivo neutralization of MCP-1, MIP- $1 \alpha$, or RANTES resulted not only in blockade of AA (Figure 1, Figure 6a, and Figure 7), but also in a marked inhibition of DTH response to PPD (Figure 6b and Figure 7c). This not only suggests that all three $\mathrm{C}$ - $\mathrm{C}$ chemokines manifest an in vivo role in the regulation of PPD-induced DTH and AA, but also that regulation of chemokine activity in vivo could not be interpreted easily using simple chemokine-chemokine receptor-output analysis.

\section{Acknowledgments}

We would like to thank H. Gershon for creative discussion and reading the manuscript, and A. Benderly for helping with the statistical analysis. This study was supported by Israel Cancer Research Foundation (ICRF), Israel Science Foundation, Israel Ministry of Science \& Arts, and Israel Ministry of Health. It was also supported by the Technion Vice President of Research Funds: the Albert Goodstein Research Fund, the Charles Krown Research Fund, the San Francisco Diabetes Research Fund, and the A\&E Blum Medical Research Fund.

1. Harris, E.D., Jr. 1990. Rheumatoid arthritis. Pathophysiology and implications for therapy. N. Engl. J. Med. 322:1277-1289.

2. Pearson, C.M. 1956. Development of arthritis, periarthritis and periostitis in rats given adjuvants. Proc. Soc. Exp. Biol. Med. 91:95-101.

3. Feldmann, M., Brennan, F.M., and Maini, N. 1996. Role of cytokines in rheumatoid arthritis. Annu. Rev. Immunol. 14:397-440.

4. Arend, W.P., et al. 1994. Binding of IL-1 alpha, IL-1 beta, and IL-1 receptor antagonist by soluble IL-1 receptors and levels of soluble IL-1 receptors in synovial fluids. J. Immunol. 153:4766-4774.

5. Arend, W.P., and Dayer, J.M. 1995. Inhibition of the production and effects of interleukin-1 and tumor necrosis factor alpha in rheumatoid arthritis. Arthritis Rheum. 38:151-160.

6. Elliott, M.J., et al. 1994. Randomised double-blind comparison of chimeric monoclonal antibody to tumour necrosis factor alpha (cA2) versus placebo in rheumatoid arthritis. Lancet. 344:1105-1110.

7. Feldmann, M., Elliott, M.J., Woody, J.N., and Maini, R.N. 1997. Antitumor necrosis factor-alpha therapy of rheumatoid arthritis. Adv. Immunol. 64:283-350.

8. Moreland, L.W., et al. 1996. Recombinant soluble tumor necrosis factor receptor (p80) fusion protein: toxicity and dose finding trial in refractory rheumatoid arthritis. J. Rheumatol. 23:1849-1855.

9. Moreland, L.W., Heck, L.W., Jr., and Koopman, W.J. 1997. Biologic agents for treating rheumatoid arthritis. Concepts and progress. Arthritis Rheum. 40:397-409.

10. Rollins, B.J. 1997. Chemokines. Blood. 90:909-928.

11. Sallusto, F., Lanzavecchia, A., and Mackay, C.R. 1998. Chemokines and chemokine receptors in T-cell priming and Th1/Th2-mediated responses. Immunol. Today. 19:568-574.

12. Ward, S.G., Bacon, K., and Westwick, J. 1998. Chemokines and T lymphocytes: more than an attraction. Immunity. 9:1-11.

13. Karpus, W.J., et al. 1995. An important role for the chemokine macrophage inflammatory protein-1 alpha in the pathogenesis of the $\mathrm{T}$ cell-mediated autoimmune disease, experimental autoimmune encephalomyelitis. J. Immunol. 155:5003-5010.

14. Gong, J.H., Ratkay, L.G., Waterfield, J.D., and Clark-Lewis, I. 1997. An antagonist of monocyte chemoattractant protein 1 (MCP-1) inhibits arthritis in the MRL-lpr mouse model. J. Exp. Med. 186:131-137.

15. Barnes, D.A., et al. 1998. Polyclonal antibody directed against human RANTES ameliorates disease in the Lewis rat adjuvant-induced arthritis model. J. Clin. Invest. 101:2910-2919.

16. Wildbaum, G., and Karin, N. 1999. Augmentation of natural immunity 
to a pro-inflammatory cytokine (TNF-a) by targeted DNA vaccine confers long lasting resistance to experimental autoimmune encephalomyelitis. Gene Ther. 6:1128-1138.

17. Youssef, S., et al. 1998. Long lasting protective immunity to experimental autoimmune encephalomyelitis following vaccination with naked DNA encoding C-C chemokines. J. Immunol. 161:3870-3879.

18. Lider, O., Karin, N., Shinitzky, M., and Cohen, I.R. 1987. Therapeutic vaccination against adjuvant arthritis using autoimmune T-cells treated with hydrostatic pressure. Proc. Natl. Acad. Sci. USA. 84:4577-4580.

19. Stein, C.S., St. Louis, J., and Strejan, G.H. 1993. Myelin-liposome protection against experimental autoimmune encephalomyelitis is associated with reduced neuroantigen-specific T-cell-mediated responses. Cell Immunol. 146:80-95.

20. Bacha, P., Forte, S.E., Perper, S.J., Trentham, D.E., and Nichols, J.C. 1992. Antiarthritic effects demonstrated by an interleukin-2 receptor-targeted cytotoxin (DAB486IL-2) in rat adjuvant arthritis. Eur. J. Immunol. 22:1673-1679.

21. Ulmer, J.B., et al. 1993. Heterologous protection against influenza by injection of DNA encoding a viral protein. Science. 259:1745-1749.

22. Tang, D.C., DeVit, M., and Johnston, S.A. 1992. Genetic immunization is a simple method for eliciting an immune response. Nature. 356:152-154.

23. Ulmer, J.B., Sadoff, J.C., and Liu, M.A. 1996. DNA vaccines. Curr. Opin. Immunol. 8:531-536.
24. Donnelly, J.J., Ulmer, J.B., and Liu, M.A. 1997. DNA vaccines. Life Sci. 60:163-172.

25. Fu, T.M., et al. 1997. Priming of cytotoxic T lymphocytes by DNA vaccines: requirement for professional antigen presenting cells and evidence for antigen transfer from myocytes. Mol. Med. 3:362-371.

26. Sato, Y., et al. 1996. Immunostimulatory DNA sequences necessary for effective intradermal gene immunization. Science. 273:352-354.

27. Raz, E., et al. 1996. Preferential induction of a Th1 immune response and inhibition of specific IgE antibody formation by plasmid DNA immunization. Proc. Natl. Acad. Sci. USA. 93:5141-5145.

28. Cyster, J.G., Hartley, S.B., and Goodnow, C.C. 1994. Competition for follicular niches excludes self-reactive cells from the recirculating B-cell repertoire. Nature. 371:389-395.

29. Youssef, S., Wildbaum, G., and Karin, N. 1999. Prevention of experimental autoimmune encephalomyelitis by MIP-1alpha and MCP-1 naked DNA vaccines. J. Autoimmun. 13:21-29.

30. Halloran, M.M., et al. 1999. The role of an epithelial neutrophil-activating peptide-78-like protein in rat adjuvant-induced arthritis. J. Immunol. 162:7492-7500.

31. Schimmer, R.C., et al. 1998. Streptococcal cell wall-induced arthritis: requirements for IL-4, IL-10, IFN-gamma, and monocyte chemoattractant protein-1. J. Immunol. 160:1466-1471. 